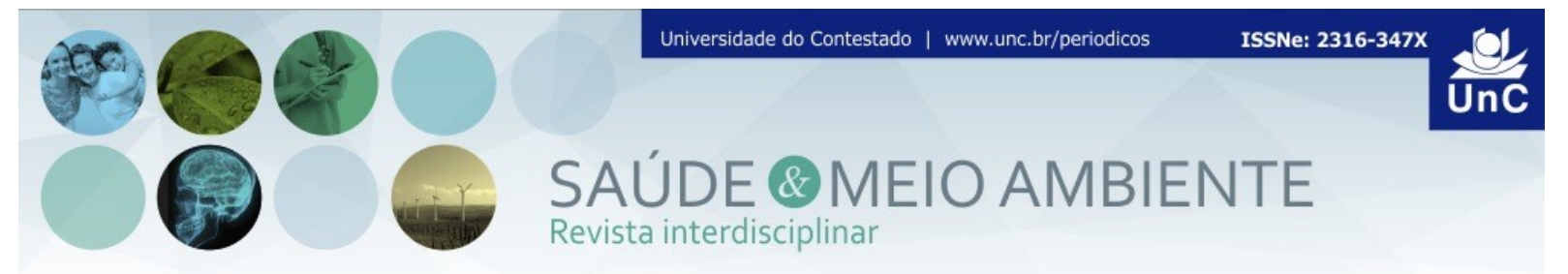

\title{
A PROMOÇÃO DA SAÚDE E A FORMAÇÃO INICIAL DO PROFISSIONAL DE SAÚDE: DESAFIOS E POSSIBILIDADES
}

\author{
Mathias Roberto Loch ${ }^{1}$
}

\begin{abstract}
RESUMO: Este texto tem como objetivo apresentar algumas reflexões sobre a formação inicial do profissional de saúde e a necessidade da aproximação desta formação com o ideário da promoção da saúde. Busca-se apontar algumas limitações do modelo biomédico e apresentar alguns pontos do ideário da promoção da saúde. São apresentadas e discutidas quatro estratégias que poderiam resultar em uma maior aproximação da formação profissional em saúde com a promoção da saúde, sendo que todas pressupõem a adoção de uma visão ampliada de saúde, a saber: necessidade de convencer e instrumentalizar professores, estudantes, trabalhadores e usuários para novas possibilidades pedagógicas e para a necessidade do trabalho interprofisisonal; garantir que aconteçam atividades de integração entre estudantes de diferentes áreas do conhecimento ao longo do curso de formação inicial; buscar uma maior articulação ensino e serviço desde o início do curso; e buscar desenvolver em todos os atores envolvidos a habilidade comunicativa, que é fundamental para o trabalho em equipe. Certamente, outras estratégias poderiam ser discutidas, mas acredita-se que a formação inicial dos cursos da área da saúde teriam importantes avanços se estas estratégias fossem efetivadas.
\end{abstract}

Palavras-chave: Formação de recursos humanos. Ensino. Formação profissional. Interdisciplinariedade.

\section{HEALTH PROMOTION AND THE FORMATION OF HEALTH PROFESSIONALS: CHALLENGES AND OPPORTUNITIES}

\begin{abstract}
This text aims to present some reflections about formation of health professionals and the need to approach this formation with the health promotion values. Some limitations of the biomedical model and some points of health promotion values are discussed. Four strategies are presented and discussed that could to be important, all of which presuppose the adoption of a broader view of health: need to convince and train teachers, students, workers and users to new pedagogical possibilities and the need for interprofessional working; ensure that integration activities take place between students from different areas of knowledge throughout the formation of students; seek greater articulation teaching and service since the beginning of the course; and seek to develop on all stakeholders to 1Doutor em Saúde Coletiva pela Universidade Estadual de Londrina, Brasil (2013). Professor Adjunto
da Universidade Estadual de Londrina, Brasil. E-mail: mathias@uel.br
\end{abstract}


communication skills, which is key to interprofessional working. Others strategies could be discussed, but it is believed that the formation of health courses have important advances if these strategies were effective.

Keywords: Human Resources. Educational Measurement. Staff Development. Interdiscisciplinarity.

\section{COMENTÁRIOS INICIAIS}

O objetivo deste texto é apresentar algumas reflexões sobre a formação inicial do profissional de saúde e a necessidade da aproximação desta formação com o ideário da promoção da saúde. Busca-se apontar e discutir algumas possíveis estratégias para uma maior aproximação da formação profissional em saúde com a promoção da saúde. Desde já, destaca-se que o sucesso destas ações pressupõe que os envolvidos apresentem uma visão ampliada de saúde. Muitos pontos do presente texto foram abordados em uma palestra com o tema: "Formação profissional em saúde" realizada durante o I Congresso Norte Catarinense Multidisciplinar em promoção à Saúde realizado no dia 16 de Outubro de 2014, na Universidade do Contestado, Campus de Mafra - Santa Catarina.

O tema é sem dúvida bastante complexo e não se tem a pretensão de esgotar o tema. Por exemplo: a leitura de outros textos que abordam a temática da formação profissional em saúde e/ou da promoção da saúde, alguns dos quais citados ao longo do texto, é fundamental. Este texto parte das seguintes premissas: 1) 0 mundo atual é extremamente complexo, com grande diversidade de valores e concepções de mundo; 2) Os grandes problemas atuais cada vez respeitam menos as rígidas fronteiras de saberes entre as áreas de conhecimento; 3) Dado este mundo complexo e as características dos principais problemas existentes, a formação em saúde deveria formar para uma melhor atuação nesta complexidade.

Não serão aprofundadas maiores questões sobre as premissas colocadas acima. Porém, para exemplificar, pensemos em alguns dos problemas que mais dizem respeito à área da saúde atualmente: demora para conseguir consultas especializadas, atendimento desumanizado, interpretação da saúde como mera mercadoria, obesidade, mortes por causas externas, problemas relacionados à saúde mental, excesso de medicalização, elevada prevalência de comportamentos de risco como inatividade física, consumo abusivo de álcool, excesso de consumo de alimentos industrializados, investimentos insuficientes para a área (sub financiamento), entre outros. Ora, parece claro que nenhuma área do conhecimento pode arrogar para si a exclusividade destes problemas. É até possível que algumas áreas considerem que tem uma maior aproximação com este ou aquele problema. Por exemplo, a nutrição, enquanto área, pode argumentar, que tem um papel central nas políticas que tratam do consumo dos alimentos industrializados. A educação física, pode considerar que deve ser a área principal quando se trata da questão da inatividade física. A psicologia pode ter um papel mais claro nas questões relativas à saúde mental, e assim por diante. Estas especificidades são importantes. Porém, 
estas áreas não podem, nem devem, abrir mão da potencial valiosa ajuda que outras áreas podem exercer na atuação sobre estes problemas específicos. Do mesmo modo, nenhuma área pode se fechar às suas especificidades mais claras, sob pena inclusive de passar a adotar estratégias simplistas e que não contemplem a complexidade da saúde. Assim, fica clara a necessidade do trabalho interprofissional, que, segundo Peduzzi et al. (2001) refere-se à integração do trabalho executado por profissionais de diversas áreas, por meio da sua interação e comunicação, preservando a especificidade do trabalho de cada área, mas pressupondo a interdependência entre estas.

Evidentemente que superar o modelo biomédico, ainda hegemônico na área da saúde, não é tarefa simples, inclusive porque este está vinculado ao modelo de organização dos cursos, que existe há muito tempo e que formou a maioria dos professores que atualmente atuam nos cursos de formação inicial da área da saúde. Não custa lembrar que qualquer mudança gera desconfia e estranhamento, e muitos não parecem dispostos a modificar suas estratégias pedagógicas, inclusive porque "naturalizaram" a organização atual, ponto que será aprofundado posteriormente neste texto.

Oliveira (2003) discute a importância do papel do profissional de saúde na formação acadêmica e menciona possíveis motivos para a não realização de mudanças nos cursos de graduação da área da saúde. Por parte das universidades, cita a gestão muitas vezes não comprometida, a atenção básica aparecendo de maneira marginal nos cursos, o despreparo dos professores frente aos novos enfoques de aprendizagem, a difícil relação entre as diferentes profissões e a resistência dos estudantes em relação ao contato com a comunidade. Já nos serviços de saúde, observam-se fatores como: a resistência dos profissionais às mudanças, o fato de a formação de outros profissionais não fazer parte da agenda de trabalho, o acréscimo de mais uma função sem remuneração para tal, a falta de estrutura física adequada, a resistência da população à presença dos estudantes no serviço e a possível identificação de fragilidades no serviço prestado.

Porém, apesar destas mudanças serem difíceis e de estas nem sempre acontecerem na velocidade desejada, há de se considerar que alguns fatos importantes acontecidos nos últimos anos, de algum modo, induzem mudanças no sentido de possibilitar uma formação mais adequada para a atuação interprofissional. A criação dos Núcleos de Apoio à Saúde da Família, a institucionalização da Promoção da Saúde no contexto do SUS (com a publicação da Política Nacional de Promoção da Saúde em 2006 e a revisão desta em 2014), o fomento as residências multiprofissionais em saúde e a programas como o PET Saúde, entre outros, são importantes neste contexto.

Para melhor organização deste texto, a seguir são apresentados alguns elementos para se refletir sobre o ideário da promoção da saúde e a necessidade de se superar o modelo biomédico. Posteriormente são apresentadas possíveis estratégias que podem contribuir para que pelo menos algumas mudanças aconteçam no sentido de fomentar o trabalho interprofissional e aproximando do ideário da promoção da saúde. 


\section{O IDEÁRIO DA PROMOÇÃO DA SAÚDE E A NECESSIDADE DE SE SUPERAR O MODELO BIOMÉDICO}

A história da saúde se confunde com a própria história da humanidade. As concepções de saúde e doença modificaram ao longo da história, acompanhando, em geral, a visão hegemônica de mundo de cada época e cultura. Para os interessados nesta temática, sugere-se a leitura de Scliar (2002; 2007). Grosso modo, podemos dizer que o olhar biomédico passa a ser hegemônico especialmente a partir das importantes contribuições de Louis Pasteur (1822-1895), que elucidou o papel das bactérias no aparecimento de doenças e de Robert Koch (1843-1910), que formulou de maneira precisa o conceito de etiologia, criando um conjunto de critérios que deveriam ser considerados para se comprovar que um micro-organismo é causador de uma doença específica. A partir daí, muito se avançou, como por exemplo, na criação de vacinas e medicamentos que foram e são utilizados para prevenir, minimizar ou até erradicar algumas doenças importantes.

Capra (2006) considera que estes exemplos servem bem para ilustrar os aspectos positivos e as limitações do olhar biomédico. O aspecto positivo é evidente, e ajudou a prevenir milhares de mortes prematuras e muito sofrimento humano. Porém, e aqui está o lado negativo, isso levou à hegemonia de uma visão limitada de saúde, que restringe os problemas de saúde aos fenômenos biológicos, passando a se buscar encontrar o mecanismo central de cada doença específica, que, uma vez encontrado passou a ser tratado por um medicamento específico, se esquecendo, ou pelo menos minimizando, os determinantes mais amplos de saúde e doença.

Como parte de resposta à esta limitação, surgiu, principalmente a partir da década de 1960, quando se ganha força a discussão sobre a determinação social da saúde, o movimento da "Promoção da Saúde". Este movimento ganha importância a partir da realização de importantes conferências, como a $1^{\mathrm{a}}$ Conferência Internacional sobre Cuidados Primários de Saúde, que se realizou em Alma-Ata, em 1978 e a $1^{a}$ Conferência Internacional de Promoção da Saúde, em 1986, em Ottawa, no Canadá. Desde então, diversos outros eventos e documentos foram realizados, e acrescentaram novos elementos à discussão da Promoção da Saúde. Além de outras sete conferências internacionais de Promoção da Saúde (a mais recente acontecida em Helsinki, 2013 e que teve como uma de suas principais recomendações a necessidade de que todas as políticas públicas, de qualquer área, considerem a questão da saúde, reforçando ainda mais uma ideia central da Promoção da saúde, que é seu caráter intersetorial, ou seja, a questão da saúde não é tema exclusivo do setor saúde. Este é mais um ponto que reforça a necessidade de uma formação mais ampliada dos profissionais de saúde), outras conferências e documentos foram elaborados, como a Estratégia Global de Alimentação Saudável, Atividade Física e Saúde (2004), o Fórum Global para o enfrentamento das doenças crônicas não transmissíveis (DCNT) em Moscou (2010), a Reunião de Alto Nível da ONU sobre DCNT em Nova York (2011), a Cúpula Mundial sobre Determinantes Sociais da Saúde no Rio de Janeiro (2011), a 
Conferência das Nações Unidas sobre Desenvolvimento Sustentável no Rio de Janeiro (2012), entre outras.

Dado este contexto, fica claro que a questão da Promoção da Saúde é complexa e pressupõe uma visão ampliada de saúde. Diga-se de passagem que é possível que alguns que utilizam a expressão muitas vezes o fazem de maneira descontextualizada e pouco criteriosa, ignorando os princípios básicos do movimento. Já em 1984, no Congresso Canadense de Saúde Pública, foram definidos alguns princípios da promoção da saúde (adaptado de Westphal, 2006): 1) Deve envolver a população como um todo, e não focalizar apenas grupos específicos; 2) As ações devem ser dirigidas sobre os diversos determinantes do processo saúde-doença (sociais, econômicos, culturais, políticos, ambientais, etc); 3) Deve buscar combinar métodos e abordagens diversas, (que devem ser complementares e não excludentes. Podem ir do nível individual ao nível macro); 4) Deve objetivar a efetiva e concreta participação social; 5) É basicamente uma atividade do campo social e da saúde e não somente serviço de saúde.

Ou seja, não se trata, de maneira alguma, de ignorar a importância dos avanços que o olhar biomédico apresentou. E mesmo as estratégias de atenção à saúde, não podem abrir mão do conhecimento biomédico. Porém, o que se precisa superar é a limitação da saúde à este olhar.

No contexto brasileiro, vale mencionar que o movimento da Promoção da Saúde constituiu base importante para o chamado Movimento da Reforma Sanitária Brasileira, que levou à criação do Sistema Único de Saúde (SUS), o que fica evidente especialmente em alguns princípios do SUS, como universalidade, integralidade, participação social e equidade. Deste então, importantes avanços no sentido de se institucionalizar a questão da Promoção da Saúde no contexto do SUS. Maiores informações sobre algumas especificidades da Promoção da Saúde no Brasil podem ser obtidas com a leitura de Buss e Carvalho (2009) e Malta et al. (2014), entre muitos outros.

Em 2006 foi publicada Política Nacional de Promoção da Saúde (2006) que foi um marco legal importante para a Promoção da Saúde no Brasil. Em 2014, a portaria $n^{\circ} 2446$ do Ministério da Saúde, de 11 de Novembro de 2014 redefine a PNPS. Rocha et al (2014) apresentam algumas informações sobre o processo de construção da redefinição da PNPS. A portaria apresenta os valores fundantes, os princípios, as diretrizes, os temas transversais, os eixos operacionais, os temas prioritários e as competências do Ministério da Saúde e das secretarias estaduais e municipais de saúde.

É possível observar que a PNPS, desde seu processo de construção até seu documento final, busca se apropriar do conceito ampliado da Promoção da Saúde. Isto é especialmente evidente pelas abrangências das estratégias propostas e dos objetivos. Por exemplo: não basta promover atividade física e alimentação saudável. É preciso que as ações que tenham este objetivo sejam coerentes com determinados valores e princípios. 


\section{A FORMAÇÃO INICIAL NAS ÁREAS DA SAÚDE: ENTRE O IDEAL E O POSSÍVEL}

O olhar biomédico influenciou claramente a organização dos cursos da área da saúde no Brasil, especialmente a partir do Relatório Flexner, publicado em 1910. Para Capra (2006) este relatório serviu como um embasamento decisivo para o ensino da medicina nos Estados Unidos, fixando diretrizes rigorosas, que passaram a dar maior ênfase ao estudo das doenças do que à assistência aos enfermos. Neste caso, ainda segundo Capra (2006), a ciência a ser ensinada e a pesquisa a ser desenvolvida nas escolas médicas estavam inseridas fortemente no contexto biomédico e tinham que ser dissociadas de preocupações sociais, que estariam fora das fronteiras da medicina. Sob o impacto do Relatório Flexner, a medicina científica voltou-se cada vez mais para a biologia e para a superespecialização.

Por outro lado, Almeida Filho (2010), faz uma importante revisita ao Relatório Flexner e considera que algumas das críticas feitas à Flexner são equivocadas. Escreve Almeida Filho (2010, p. 2247):

[...] equivocam-se os que consideram Flexner como defensor de uma formação com base em conteúdos e não em métodos, que sua visão de saúde era exclusivamente biológica e reducionista, e não social e humanista, que a vertente principal do seu modelo de prática é a da medicina curativa e individual, e que Flexner apoiava a prática privada da medicina e o controle social da formação médica pelas corporações profissionais. Porém, estão corretos os que acham que ele propunha uma reorganização do conhecimento com base numa abordagem disciplinar [...].

Almeida Filho (2010) ainda aponta trechos do relatório em que Flexner defende que os médicos deveriam ter uma educação geral, incluindo uma boa formação nas ciências humanas e sociais, antes mesmo da formação nas ciências básicas da saúde.

Pelo visto, nem todos os autores apresentam a mesma interpretação do Relatório Flexner. O que fica claro é a influência, mesmo que talvez a partir de interpretações equivocadas de alguns pontos, deste relatório na organização dos cursos da área da saúde, em um primeiro momento na medicina e posteriormente à outras áreas. Esta influência está presente até os dias de hoje.

Hallal (2011, p. 92), abordando a criação do Programa Academia da Saúde, pelo Ministério da Saúde, menciona a necessidade de se rediscutir com urgência a formação inicial na área da saúde. O autor escreve:

Pergunte a um estudante de medicina como tratar um paciente com
diabetes descompensada e é muito provável que você receba uma resposta
correta ou algo muito próximo disso. Agora pergunte ao mesmo estudante
como reduzir a incidência de diabetes no nível populacional. Silêncio. Faça
o mesmo com um acadêmico de nutrição. Peça a ele para montar uma dieta
para um paciente obeso. Dieta em mãos. Depois peça para ele montar um
plano de prevenção da obesidade no estado onde ele vive. Silêncio. Repita
o exercício para um profissional de educação física. Primeiro a prescrição
de um programa de exercícios para um paciente cardiopata. Feito. Depois a 
montagem de uma estratégia para tornar a população brasileira mais ativa. Silêncio.

No texto citado, fica claro que, para o autor, a formação na área da saúde é hegemonicamente centrada nos seus aspectos clínicos. Claro que podemos argumentar um certo exagero (proposital certamente) do autor e até citarmos alguns (poucos) exemplos que desmentiriam as colocações do autor. Porém, em linhas gerais, parece que o texto capta muito bem a realidade atual. Quando muito, formamos bons profissionais para a atuação no contexto da orientação/prescrição individualizada.

Além disso, um outro ponto a ser destacado é que a formação de cada área da saúde se dá de maneira isolada das outras. Andrade et al (2014), falando especificamente da Educação Física, mencionam que boa parte da formação do profissional desta área acontece em uma lógica de atuação profissional individual. Esta é possivelmente a realidade hegemônica da maioria, se não de todas, as áreas da saúde no Brasil. Além disso, mesmo dentro das próprias áreas existem poucas experiências interdisciplinares. Cada disciplina cumpre a sua função de maneira isolada e acredita-se que cada aluno terá a capacidade de "juntar as partes" na sua futura atuação profissional.

Esta realidade (formação hegemonicamente centrada na atuação clínica e individualizada na própria profissão) está em desacordo com as atuais necessidades de saúde e com as políticas públicas de saúde, como mencionado na introdução deste texto, o que leva à uma situação complexa, pois aquilo que é necessário e exigido, não é o que a maior parte dos cursos de formação inicial da área da saúde consegue oferecer, estando muito distante do ideário da Promoção da Saúde.

De qualquer modo, parece claro que mudanças são necessárias. Inclusive por um motivo mais "prático" do que os apresentados até agora neste texto: o próprio perfil dos alunos. A geração atual, e certamente as que virão, parece ter uma capacidade de concentração reduzida se comparada às gerações anteriores. Por outro lado, a quantidade de informações e a forma, bem mais ampliada, de se obtêlas é algo a ser destacado. A aula não é mais a única possibilidade de se "adquirir" conhecimento. Assim, não é concebível que imaginemos que um professor possa dar aula da mesma maneira que dava 10 ou 15 anos atrás. Em outras palavras: as estratégias de ensino precisam se adaptar também, mas não exclusivamente é claro, as necessidades dos alunos.

Mesmo assim, seria ingênuo imaginar que mudanças radicais podem acontecer na formação profissional em um curto espaço de tempo. A seguir, são apresentadas possíveis estratégias que podem ser úteis no sentido de possibilitar aos alunos dos cursos de formação inicial na área da saúde uma formação mais ampliada e próxima do ideário da Promoção da Saúde. 


\section{SUGESTÕES PARA A FORMAÇÃO INICIAL DE PROFISSIONAIS DA ÁREA DA SAÚDE}

1 - Necessidade de convencer e instrumentalizar professores, estudantes, trabalhadores e usuários para novas possibilidades pedagógicas e para a necessidade do trabalho interprofisisonal.

Talvez este seja um ponto crucial. Mais uma vez, há de se destacar que esta modificação não se dará da noite para o dia. Ela é (uma vez que já está em curso) um processo, e talvez leve décadas para que um olhar ampliado de saúde seja hegemônico em relação ao olhar biomédico. Ou talvez, nunca seja hegemônico. De qualquer modo, algo precisa ser feito para se aumentar a proporção de profissionais de saúde que tenham um olhar ampliado de saúde e que consigam atuar profissionalmente colocando este olhar em prática.

Parece que atualmente muitos professores dos cursos de formação inicial da área da saúde já passaram a perceber um certo esgotamento do modelo tradicional de organização curricular e mesmo das estratégias pedagógicas centradas no professor e com caráter meramente conteudista, ou seja, que se preocupa mais com a grande quantidade de conteúdos e menos com o desenvolvimento intelectual, cultural e de raciocínio dos alunos. É verdade que outros professores parecem ter ainda grande convicção neste modelo tradicional. De fato, superar a noção de que o conhecimento se dá em "caixinhas" não é tarefa fácil. Quase todos os professores universitários que atuam atualmente foram formados nessa lógica. Assim, muitos naturalizaram que o processo ensino aprendizagem precisa ser desta forma, pois é assim que eles aprenderam. Não se trata de negar que é verdade que se aprendeu desta forma durante muitas décadas. Porém, há de se considerar as mudanças ocorridas na sociedade como um todo, nos problemas de saúde, no perfil dos alunos, na possibilidade de se obter conhecimento através de formas que eram impossíveis décadas atrás, etc. Como mencionado na primeira premissa deste texto: o mundo se modificou e ficou mais complexo. Não é possível mais ignorar este fato. A educação, em todos os níveis, precisa preparar para esta complexidade, contribuindo inclusive para que o mundo se torne mais justo, tolerante e solidário. Neste sentido, a superação do olhar biomédico e a maior aproximação com o ideário da promoção da saúde seria importante.

Por parte dos estudantes dos cursos de formação inicial, esta tarefa é, possivelmente, menos complexa. Não que seja fácil, já que a maioria dos alunos ainda são formados no nível fundamental e médio nesta lógica do conhecimento em "caixinhas". Porém, em geral, parece que estão mais abertos à, pelo menos, experimentarem novas possibilidades pedagógicas. Como já mencionado, muitos sequer tem capacidade de concentração para "assistirem" à uma aula tradicional de duas horas, por exemplo. E não se trata de culpar os alunos por esta "falta de capacidade". O mundo em que eles foram formados é bem mais dinâmico do que os de décadas posteriores. A maioria já cresceu com acesso fácil à internet, para ficar apenas em um exemplo. Só isso já representaria uma mudança enorme no perfil. Assim, estratégias mais dinâmicas e desafiadoras costumam ser bem recebidas 
pelos alunos. Assim, é importante que os alunos sejam protagonistas do seu próprio ensino e não apenas meros receptores do conhecimento.

Um ponto posterior irá tratar da importância de uma maior articulação com os serviços de saúde. Para isso, também é necessário que estes serviços sejam convencidos e instrumentalizados. Nada adianta a universidade se modificar e o serviço que recebe os alunos para seus estágios e outras atividades de ensino, pesquisa e extensão, estarem limitados ao olhar biomédico. Logicamente, esta mudança se dará com o próprio processo. Não dá para simplesmente esperar que o serviço esteja "convencido e instrumentalizado" para fazer a inserção dos alunos. A própria inserção deve, de alguma maneira, ser indutora de mudanças. Domingues (2012) estudou especificamente o discurso dos trabalhadores de Unidades Básicas de Saúde (UBS) que haviam recebido alunos do curso de Residência Multiprofissional em Saúde da Família. Entre os achados da autora, ela observou que muitos trabalhadores das UBS relatavam um grande estranhamento no momento de inserção dos residentes, especialmente daqueles que eram de áreas não habituais neste local de trabalho. Assistentes sociais, profissionais de educação física, nutricionistas, entre outros, eram visto com certa desconfiança, pois muitos não tinham ideia do que estes profissionais poderiam fazer em uma UBS. Algum tempo depois, já havia um processo de naturalização da presença destes novos profissionais. Inclusive alguns profissionais das UBS já tinham dificuldade de imaginar a unidade sem a presença destas novas profissões, principalmente porque a quantidade e qualidade das ofertas dos serviços das UBS aumentou, possibilitando assim uma maior aproximação com um olhar ampliado de saúde. Outro resultado importante do estudo de Domingues (2012) é que os profissionais das UBS percebiam que a presença dos residentes era indutora de mudanças positivas no processo de trabalho, como por exemplo, no fomento à discussões de casos, no encaminhamento para ações de educação em saúde, entre outras. É lógico que nenhum processo como este é livre de conflitos e resistências. Porém, as evidencias apontadas pela autora mostram que o saldo pode ser bastante positivo.

Quanto aos usuários, estes também devem ser considerados neste processo. Não são meros receptores dos cuidados de saúde. Alguns usurários também parecem ter naturalizado o olhar biomédico como o único possível nos serviços de saúde. Estes, quando procuram algum serviço parecem acreditar que o único profissional existente é o médico. E quando consultados por estes, esperam que sejam encaminhados à exames complexos e recebem a indicação de medicamentos. Do contrário, a consulta é muitas vezes avaliada como de baixa qualidade. Esta é uma situação que reflete a visão de saúde que é hegemônica na nossa sociedade, tendo uma forte relação com o que alguns autores vão denominar "medicalização da vida". Diga-se de passagem que a formação profissional centrada no olhar biomédico reforça esta visão e contribui muito para que esta seja a visão de muitos usuários. Descontruir isso não é simples, mas sem dúvida é necessário. Ações como o fomento de atividades de educação em saúde, estimulo à atividade de participação e controle social, além de ações ainda mais amplas como a melhor regulamentação das propagandas de medicamento, poderiam ser importantes. 
2 - Garantir que aconteçam nos cursos de formação inicial, atividades de integração entre estudantes de diferentes áreas do conhecimento.

Sem dúvida exigir que o profissional de saúde saiba trabalhar em grupo sem que este tenha sido preparado para tal, é algo problemático. Verdade que muitos profissionais, mesmo sem esta habilidade desenvolvida nos cursos de formação inicial, acabam aprendendo ao longo do processo. As atividades de educação permanente são fundamentais. Porém, é muito provável que haja uma melhor atuação interprofissional se os estudantes tiverem uma formação mais específica para tal.

Muitas vezes a própria estrutura universitária acaba dificultando estas ações. A maioria das universidades brasileiras se organiza em departamentos e colegiados que, em geral, pouco dialogam com outros departamentos e cursos. Idealmente, as atividades entre os cursos deveriam acontecer de maneira bem mais orgânica, mas esta é uma realidade que parece distante para a maioria dos cursos. Para pelo menos minimizar este distanciamento, é preciso se garantir pelo menos algumas ações específicas. Alguns exemplos: disciplinas e/ou módulos específicos, projetos de pesquisa, ensino e extensão, visitas aos diferentes serviços de saúde, os estágios nos serviços, estímulo à participação em congressos que tem a participação de diferentes áreas, etc. É possível que todos, ou quase todos, os cursos da área da saúde tenham um módulo e/ou disciplina que trata da epidemiologia geral ou da organização do serviço de saúde. Talvez fosse possível em algumas universidades se "misturar" os alunos, de modo que ao invés de, por exemplo, termos cinco diferentes cursos trabalhando o mesmo conteúdo isoladamente, teríamos cinco turmas, em todas elas com alunos de todos os cinco cursos, trabalhando este conteúdo. Para que aconteça uma integração dos alunos também é necessário que existam espaços em comum para os cursos. Por exemplo: um ou dois períodos na semana sem aulas formais mas protegidos para participação em atividades em projetos de pesquisa, extensão ou ensino com caráter interdisciplinar.

Um possível efeito desta melhor integração seria possibilitar um melhor conhecimento das características de cada área. É provável que atualmente a maior parte dos alunos dos cursos da área da saúde pouco saiba sobre as possibilidades de atuação de seus colegas de outras profissões, prevalecendo uma visão de senso comum, que muitas vezes meramente reproduz alguns estereótipos.

Esta realidade de que há pouco contato entre os diferentes cursos da área da saúde durante a graduação não é exclusividade brasileira. Esta é uma das conclusões do estudo de Coster et al. (2008) no Reino Unido. Outro resultado importante deste estudo aponta que o interesse e a prontidão para o ensino interprofissional é alto no início e diminui no decorrer da formação para todas as áreas, à exceção da enfermagem. A partir do momento em que começam a ter uma formação isolada na sua própria área de atuação, muitos alunos podem sentir inclusive rejeição à atividades interdisciplinares e interprofissionais. 


\section{3 - Buscar uma maior articulação ensino e serviço desde o início do curso.}

Muitos cursos ainda se organizam na lógica: primeiros anos do curso de "teoria" e últimos anos de "prática", estas principalmente a partir dos estágios. Porém, esta lógica parece estar muito mais favorecendo um distanciamento entre "teoria" e "prática" do que contribuindo para uma melhor formação.

É preciso se destacar que os serviços de saúde devem também ser espaços de aprendizagem. Um dos aspectos positivos desta aproximação mais precoce com os serviços é enfatizar uma aprendizagem que seja significativa, ou seja, que promova e produza sentidos, e que busquem induzir a transformação das práticas profissionais que sejam baseadas na reflexão crítica sobre as práticas reais (Ceccim e Feuerwerker, 2004).

Esta maior aproximação deve inclusive possibilitar a reflexão, que deve ser permanente, sobre o papel da universidade. Mello, Almeida Filho e Ribeiro (sem data) escrevem sobre a necessidade de a Universidade ter um papel relevante, inclusive equilibrando a pertinência científica com a relevância social. Para os autores, há a necessidade de uma maior inserção das Universidades nos seus contextos locais, pois a comunidade $e$ as instituições são ambientes de aprendizagem pedagogicamente promissoras, propícias à reflexão crítica sobre a realidade, enquanto a sala de aula, isoladamente, não oferece possibilidade real de passagem da informação para o conhecimento, nem do conhecimento para a sabedoria. Assim, fica claro que a formação deve ir para além da questão instrumental ou técnica do conhecimento, e a criação de uma relação mais orgânica entre universidade e comunidade se faz necessária.

4 - Buscar desenvolver em todos os atores envolvidos a habilidade comunicativa, que é fundamental para o trabalho em equipe.

Assumir que acontecem conflitos em qualquer trabalho em equipe é necessário. Na verdade, na maioria das vezes, espaços sem conflitos são espaços com grande assimetria de poder, o que é indesejável para que o trabalho interprofissional possa de fato acontecer com qualidade.

Para se minimizar e aprender a se lidar com estes conflitos, o desenvolvimento de habilidades comunicativas se faz necessário. Cada ator envolvido neste processo precisa ter habilidade para falar e escutar. Deve-se buscar a construção de relações mais horizontais.

Mais uma vez fica clara a necessidade de que a formação em saúde vá para além do aspecto técnico e procedimental. Ademais, vale destacar que existem diferentes tipos de trabalho em equipe. Na lógica do "agrupamento" acontece a justaposição das ações e mero agrupamento dos profissionais, a interação/comunicação entre os profissionais acontece com ênfase na transmissão de informações, a liderança centralizada, ou seja, existe uma gestão hierárquica, a responsabilidade é individual, bem como os resultados e as metas. Já na lógica da integração, acontece uma maior articulação das ações e interação dos profissionais, 
a Interação/comunicação entre os profissionais se dá com ênfase no diálogo, a liderança é partilhada, ou seja, à co-gestão, as responsabilidades são individuais e coletivas e a avaliação dos resultados é coletivo, havendo metas específicas da equipe, com objetivos comuns definidos e metas comuns do serviço (Peduzzi et al., 2001). Fica assim evidente que a lógica da "integração" e que tem maior relação com o conceito de interprofissionalidade, é que deve ser buscado.

\section{COMENTÁRIOS FINAIS}

Este texto buscou argumentar favoravelmente à necessidade de algumas modificações na formação inicial nos cursos da área da saúde, com vistas à superação do modelo biomédico e uma maior aproximação com o ideário da promoção da saúde, apresentando e discutindo algumas possíveis estratégias para que estas mudanças aconteçam. Evidentemente o tema é complexo e não se buscou aqui esgotar a temática.

Considerando que o movimento da Promoção da Saúde tem uma história de influência e que é também influenciado pelo SUS no Brasil, uma maior aproximação com o SUS, especialmente com seus princípios, seria já um avanço importante para muitos cursos. Importante salientar que uma maior aproximação com o SUS, e consequentemente com o ideário da Promoção da Saúde, pode ser relevante para a melhoria da formação do profissional de saúde não apenas para aqueles que irão atuar diretamente no SUS, mas também para aqueles que irão atuar no setor privado. Isso porque o SUS apregoa uma mudança de modelo assistencial, que dá maior ênfase à aspectos como o cuidado humanizado e enfoca a integralidade em detrimento ao modelo biomédico centrado especialmente nas especialidades. Esta mudança de enfoque certamente é importante para o sistema como um todo, e não apenas para o setor público.

Dado que as necessidades de saúde da população são amplas e complexas, o trabalho interprofissional deve ser muito enfatizado. A formação inicial dos cursos da saúde precisa assumir com urgência a tarefa de possibilitar uma melhor formação de seus alunos para que estes possam melhor exercer futuramente o trabalho interprofissional.

Vale destacar que não é possível se fazer a transição de modelo sem uma certa angústia e ansiedade. O sentimento de ter que "trocar o pneu com o carro andando" estará necessariamente presente, pois não é possível "fecharmos" as universidades e os serviços de saúde, nos capacitamos e depois reabrirmos estes espaços. A mudança deverá ser feita ao longo do próprio processo, que é complexo e não linear, ou seja, é passível de avanços e retrocessos. De qualquer modo, o desafio está posto e seu enfrentamento se faz urgente. 


\section{REFERÊNCIAS}

ALMEIDA FILHO, N. Reconhecer Flexner: inquérito sobre produção de mitos na educação médica no Brasil contemporâneo. Cad. Saúde Pública, v. 26, n. 12, p. 2234-2249, 2010.

ANDRADE, D.R. et al. Formação do bacharel em educação física frente à situação de saúde no Brasil. In: BENEDETTI, T.R.B. et al. A formação do profissional de Educação Física para o setor saúde. Florianópolis: Editora Postmix, 2014.

BRASIL. Portaria n 2446, de 11 de novembro de 2014. Redefine a Política Nacional de Promoção da Saúde (PNPS). Diário Oficial da União, 2014.

BUSS, P.M.; CARVALHO, A.I. Desenvolvimento da promoção da saúde no Brasil nos últimos vinte anos (1988-2008). Ciênc. saúde coletiva, v. 14, n. 6, p. 23052316, 2009.

COSTER, S., et al. Interprofessional atitudes amongs undergraduate students in the health professions: a longitudinal questionnaire survey. International Journal of Nursing Studies, v. 45, p. 1667-81, 2008.

CAPRA, F. O ponto de mutação. São Paulo: Cultrix, 2006.

CECCIM, R. B.; FEUERWERKER, L. C. M. O quadrilátero da formação para a área da saúde: ensino, gestão, atenção e controle social. Physis, v. 14, n. 1, p. 41-65, 2004.

DOMINGUES, C. M. Residência multiprofissional em saúde da família: o olhar do trabalhador de saúde. Londrina, Paraná. 2012. Dissertação (Mestrado em Saúde Coletiva) - Universidade Estadual de Londrina, Paraná, 2012.

HALLLAL, P. C. Academia da saúde: mais um passo na direção certa. Revista Brasileira de Atividade Física e Saúde, v. 16, n. 2, p. 92, 2011.

MALTA, D.C., et al. A implementação das prioridades da Política Nacional de Promoção da Saúde, um balanço, 2006 a 2014. Ciênc. saúde coletiva, v. 19, n. 11, p. 4301-4312, 2014.

MELLO, A.F.; ALMEIDA FILHO, N., RIBEIRO, R.J. Por uma universidade socialmente relevante. Disponível em: <http://portal.mec.gov.br/dmdocuments/ cne_alexfiuza.pdf>. Acesso em: 09 jun. 2015.

PEDUZZI, M. Equipe multiprofissional de saúde: conceito e tipologia. Revista de Saúde Pública. v. 35, n. 1, p. 103-109, 2001.

OLIVEIRA, M.S. O papel dos profissionais de saúde na formação acadêmica.

Revista Olho Mágico, v. 10, n. 2, p. 37-39, 2003. 
ROCHA, D.G. et al. Processo de revisão da Política Nacional de Promoção da Saúde: múltiplos movimentos simultâneos. Ciênc. saúde coletiva, v. 19, n. 11, p. 4313-4322, 2014.

SCLIAR, M. Do mágico ao social: a trajetória da saúde pública. São Paulo: Ed. Senac, 2002.

SCLIAR, M. História do conceito de saúde. Physis, v. 17, n. 1, p. 29-41, 2007.

WESTPHAL, MF. Promoção da Saúde e prevenção de doenças. In: GASTÃO, W. S.C. et al. Tratado de saúde coletiva. Rio de Janeiro: Ed. Fiocruz, 2006.

Artigo recebido em: 11/06/2015

Artigo aprovado em: 03/07/2015 